\title{
QCD equation of state: Physical quark masses and asymptotic temperatures
}

\author{
M. Bluhm ${ }^{1}$, B. Kämpfer ${ }^{1,2}$, R. Schulze ${ }^{1}$, D. Seipt ${ }^{1}$ \\ ${ }^{1}$ Forschungszentrum Dresden-Rossendorf, PF 510119, 01314 Dresden, Germany \\ ${ }^{2}$ Institut für Theoretische Physik, TU Dresden, 01062 Dresden, Germany
}

\begin{abstract}
Within a phenomenological quasiparticle model, the quark mass and temperature dependence of the QCD equation of state is discussed and compared with lattice QCD results. Different approximations for the quasiparticle dispersion relations are employed, scaling properties of the equation of state with quark mass and deconfinement temperature are investigated and a continuation to asymptotically large temperatures is presented.
\end{abstract}

\section{Introduction}

Within the past years, physicists aimed at revealing the very nature of strongly interacting matter experimentally by ultra-relativistic heavy-ion collisions performed at CERN-SPS and BNL-RHIC (see e. g. [1, 2]). At the large local energy densities reached during the collision process, a new state of deconfined matter, dubbed quark-gluon plasma (QGP), is thought to be created. Originally, motivated by asymptotic freedom in QCD, the QGP was considered as a weakly interacting gas of quarks $(q)$ and gluons $(g)$. However, the success of hydrodynamical concepts (supplemented by assuming fast thermalization [3, 4, 5, 6] and low viscosity [7, 8, 9]) in describing experimental results points to the necessity of viewing the QGP rather as a strongly coupled system.

For hydrodynamic considerations, the QCD equation of state (EoS), which is related to the grand potential $\Omega$, is of paramount interest. In addition, the EoS is of significant importance for the description of the dynamics of the early universe or of compact stellar objects. In general, $\Omega$ depends on parameters being specific for QCD like quark masses $m_{q}$, flavor number $N_{f}$ or color number $N_{c}$ as well as on external conditions described by temperature $T$ and various chemical potentials $\left\{\mu_{q}\right\}$. From the theoretical side, much progress has been achieved in first-principle (lattice) QCD evaluations of the grand potential. Previously, the latter were technically restricted to too large $m_{q}$ values of $\mathcal{O}(T)$ translating into a pion mass of about $770 \mathrm{MeV}$ [10] at the deconfinement critical temperature $T_{c}$. Only recently, smaller quark mass parameters were considered [11] pushing the pion mass to about $215 \mathrm{MeV}$ [12, 13]. Nonetheless, despite the necessary continuum extrapolation of lattice QCD results, also an extrapolation to physical quark masses remains an inevitable step towards obtaining reliable results.

A variety of phenomenological approaches describes fairly well bulk information found in lattice QCD calculations by adjusting suitable parameters. Among these, effective quasiparticle models [14, 15, 16, 17, 18, 19], PNJL models [20, 21, 22], (Polyakov-) quark-meson models [23, 24] and a colored bound state model [25, 26] have to be mentioned. Here, we employ a quasiparticle model (QPM) for the description of QCD thermodynamics. The quark mass dependence is directly implemented in the quasiparticle 
dispersion relations, which allows for a comparison with lattice QCD results employing different quark mass values and enables an extrapolation to the physical limit. The quark mass dependence of QCD excitations in one-loop approximation was investigated in [27]. Armed by dispersion relations motivated from these considerations, the quark mass dependence of $\Omega$ is also discussed here.

Soon, even larger local energy densities may be reached at CERN-LHC, which might provide deeper insights into the early formation dynamics of our universe. For pure SU(3) gauge theory, lattice QCD thermodynamics was recently studied up to temperatures $\sim 10^{7} T_{c}[28$. At such asymptotically large temperatures, analytical attempts based on perturbative means [29, 30] account fairly well for available lattice QCD results. Here, we discuss the EoS at asymptotically large temperatures within our QPM, both, for pure $\mathrm{SU}(3)$ and for $N_{f}=2+1$. (Nevertheless, for early universe studies also the influence of the heavy quark sector becomes important [31].)

\section{Quasiparticle dispersion relations}

In the following, we concentrate on matter anti-matter symmetric systems, represented by a net baryon density $n_{B}=0$. In this case, the QPM rests on the entropy density $s=\sum_{i=g, q} s_{i}$ with

$$
s_{i}(T)=2 \epsilon_{i} \frac{d_{i}}{\pi^{2}} \int_{0}^{\infty} d k k^{2}\left(\ln \left[1+\epsilon_{i} e^{-\omega_{i} / T}\right]+\epsilon_{i} \frac{\omega_{i} / T}{e^{\omega_{i} / T}+\epsilon_{i}}\right),
$$

where $d_{q}=N_{f} N_{c}, d_{g}=N_{c}^{2}-1, \epsilon_{q}=1, \epsilon_{g}=-1$ (for details cf. [15, 32]). This ansatz assumes that the thermodynamically relevant excitations at momenta $k \sim T$ are transverse gluons and regular quark modes. The pressure $p=-\Omega / V$ ( $V$ is the volume) and other related thermodynamic quantities follow from integrating $s=d p / d T$. The quasiparticle dispersion relations entering Eq. (1) can be represented by $\omega_{g, q}^{2}=k^{2}+\Pi_{g, q}(T)$, where $\Pi_{g, q}(T)$ denote temperature dependent self-energies. The self-energies can phenomenologically be approximated by

$$
\begin{aligned}
\Pi_{g} & =\frac{1}{6} G^{2} T^{2}\left(N_{c}+\frac{N_{f}}{2}\right), \\
\Pi_{q} & =m_{q}^{2}+2 m_{f}^{2}+\alpha m_{q} m_{f}
\end{aligned}
$$

with $m_{f}^{2}=\left(N_{c}^{2}-1\right) G^{2} T^{2} /\left(16 N_{c}\right)$. These expressions (cf. [15, 32] for $\alpha=2$ ) are based on one-loop approximations to lowest order in $m_{q}$ in the asymptotic momentum region, where Eq. (3) is supplemented by a bi-linear term $\alpha m_{q} m_{f}$ for including nonzero quark masses according to [33]. $G^{2}$, replacing the QCD running coupling $g^{2}$, represents an effective coupling strength parametrized by

$$
G^{2}(T)=\left\{\begin{array}{l}
G_{(2-\text { loop })}^{2}(\zeta(T)), \quad T \geq T_{c}, \\
G_{(2-\text { loop })}^{2}\left(\zeta\left(T_{c}\right)\right)+b\left(1-\frac{T}{T_{c}}\right)+a\left(1-\frac{T}{T_{c}}\right)^{2}, T<T_{c}
\end{array}\right.
$$

with $\zeta(T)=\lambda\left(T-T_{s}\right) / T_{c}$ which approaches the perturbative region at large $T$ in line with the two-loop expression of $g^{2}$. In $\zeta(T), \lambda$ can be related to $\Lambda_{\mathrm{QCD}}$ while $T_{s}$ regulates $G^{2}$ near $T_{c}$. For $T<T_{c}, G^{2}$ changes drastically its behavior as dictated by lattice QCD results.

Recently [27, the quark mass dependence of thermal QCD excitations in the one-loop approximation was examined in some detail. The gauge invariant expression for the transverse gluon self-energy at asymptotic momenta was found as

$$
\begin{aligned}
\Pi_{g} & =\frac{1}{6} G^{2} T^{2}\left(N_{c}+\frac{1}{2} \sum_{q=1}^{N_{f}} \mathcal{I}\left(\frac{m_{q}}{T}\right)\right) \\
\mathcal{I}\left(m_{q} / T\right) & =\frac{12}{\pi^{2}}\left(\frac{m_{q}}{T}\right)^{2} \int_{0}^{\infty} d \sigma \frac{\sigma^{2}}{\sqrt{1+\sigma^{2}}} \frac{1}{\left(1+e^{\sqrt{1+\sigma^{2}} m_{q} / T}\right)} .
\end{aligned}
$$


The power expansion of $\mathcal{I}\left(m_{q} / T\right)$ for small $m_{q} / T$ involves a term resembling chiral logarithms, cf. [27]. Neglecting any quark mass dependence by setting $m_{q} / T \rightarrow 0$, one gets $\mathcal{I}\left(m_{q} / T\right) \rightarrow 1$ and thus Eq. (2) is reproduced. This approximation is supported to some extent by the one-loop results [27], as the energy of transverse gluon excitations increases only by a tiny amount when decreasing $m_{q}$.

In the case of regular quark excitations, $\Pi_{q}=m_{q}^{2}+m_{f}^{2} \mathcal{F}_{q}\left(k, m_{q}\right)$ is found in [27], where $\mathcal{F}_{q}$ (depending non-trivially on $k$ and $m_{q}$ ) encodes the effects induced by the thermal medium. Straightforward evaluation of this expression in the asymptotic momentum region yields $\Pi_{q}=m_{q}^{2}+2 M_{+}^{2}$ with $M_{+}^{2}=\frac{1}{3} m_{f}^{2}\left(\mathcal{I}\left(m_{q} / T\right)+2\right)$ and $\mathcal{I}\left(m_{q} / T\right)$ given in Eq. (66). Thus, in the limit $m_{q} \rightarrow 0, M_{+}^{2}$ reduces to $m_{f}^{2}$. Obviously and in contrast to Eq. (3), a bi-linear term relating $m_{q}$ and $m_{f}$ is not present in this result. However, for momenta $k \sim T$ and small $m_{q}$, a bi-linear term can be motivated from the one-loop approximations. In general, one can represent $\mathcal{F}_{q}\left(k, m_{q}\right)=\alpha\left(k, m_{q}\right) \mathcal{F}_{q}\left(k=0, m_{q}\right)$, where, for small $m_{q}$ (and fixed $k$ ), $\alpha$ is rather independent of $m_{q}$ and a number between 1 and 2. Approximating $\mathcal{F}_{q}\left(k=0, m_{q}\right)$ for small but nonzero $m_{q}$ and small $g$ in line with a generalization of [33],

$$
\Pi_{q}=m_{q}^{2}+\alpha\left(-\frac{1}{2} m_{q}^{2}+m_{q} M_{+}+M_{+}^{2}\right)
$$

is obtained for an approximation of the asymptotic quark self-energy. With this ansatz, the thermal quark mass effectively decreases with decreasing quark mass parameter, as in the case of Eq. (3). In fact, both approximations Eq. (3) and Eq. (7) yield the same expression for the quark self-energy in the limit $m \ll 1$ and for $\alpha=2$ reading $\Pi_{q}=2 m_{q} m_{f}+2 m_{f}^{2}$.

\section{Quark mass extrapolation}

Benchmark of the considerations is the scaled entropy density $s / T^{3}$ as a function of $T / T_{c}$ for $N_{f}=2+1$. In [10], a continuum estimate for $s / T^{3}$ is given by reporting lattice QCD results for the energy density $e$ and the interaction measure $\Delta \equiv(e-3 p)$, where $s / T^{3}=(4 e-\Delta) /\left(3 T^{4}\right)$, for fairly large and temperature dependent quark mass parameters $m_{u, d} / T=0.4$ and $m_{s} / T=1$. Recently [11, 12], $s / T^{3}$ was calculated for much smaller quark masses. Corresponding to [34], the quark mass parameters used in [11, 12] can be approximated by $m_{u} / T=m_{d} / T=A_{0} / T^{2}+A_{1} / T+A_{2}$ with $A_{0}=0.298 \cdot 10^{-3} \mathrm{GeV}^{2}, A_{1}=1.664 \cdot 10^{-3}$ $\mathrm{GeV}$ and $A_{2}=0.002$ for $T$ given in $\mathrm{GeV}$ and $m_{s}=10 m_{u}$. As $s / T^{3}$ in [12] is given as a function of $T$ in $\mathrm{MeV}$, we scale by $T_{c}=190 \mathrm{MeV}$ in line with [12], where $T_{c}=(190 \pm 5) \mathrm{MeV}$ is reported giving rise to an estimated error in $T / T_{c}$ according to $\Delta T_{c}=5 \mathrm{MeV}$. Despite, $T_{c}$ is assumed to be approximately quark (and related pion) mass independent (cf. [35] and references therein).

In the following, the QPM armed by the two different approximations for the dispersion relations discussed in Sec. 2 is adjusted to the lattice QCD results from [10]. The application of Eqs. (2) and (3) in the quasiparticle dispersion relations is denoted by "Fit 1", while the use of Eqs. (5)-(7) is associated with "Fit 2". Then, by extrapolating to the mass set-up employed in [12, the proper implementation of the quark mass dependence in the QPM can directly be tested. For simplicity, any conceivable $m_{q}$ dependence in $G^{2}$ is naively neglected.

Starting with Fit 1, i. e. Eqs. (21) and (3) as approximation of the self-energies, we choose $\alpha=1$, here, as reasonable value (cf. Sec. 2). The corresponding QPM parameters of $G^{2}$ read $T_{s}=0.7 T_{c}, \lambda=5$, $a=-426, b=403.3$ describing $s / T^{3}$ from [10] impressively well as exhibited by the lower dashed curve in Fig. 11 (left panel). Extrapolating to smaller quark masses as used in [12], the corresponding lattice QCD results are fairly well reproduced, cf. upper dashed curve in Fig. 1 (left panel). Decreasing $m_{q}$, the thermal gluon mass remains unaffected, while the thermal quark mass is reduced according to Eq. (3). Thus, the entropy density increases with decreasing quark mass.

Note that an inclusion of the term $\alpha m_{q} m_{f}$ of significant strength seems to be mandatory. Neglecting this contribution by setting $\alpha=0$, any adjustment of QPM parameters to [10] fails in describing the 

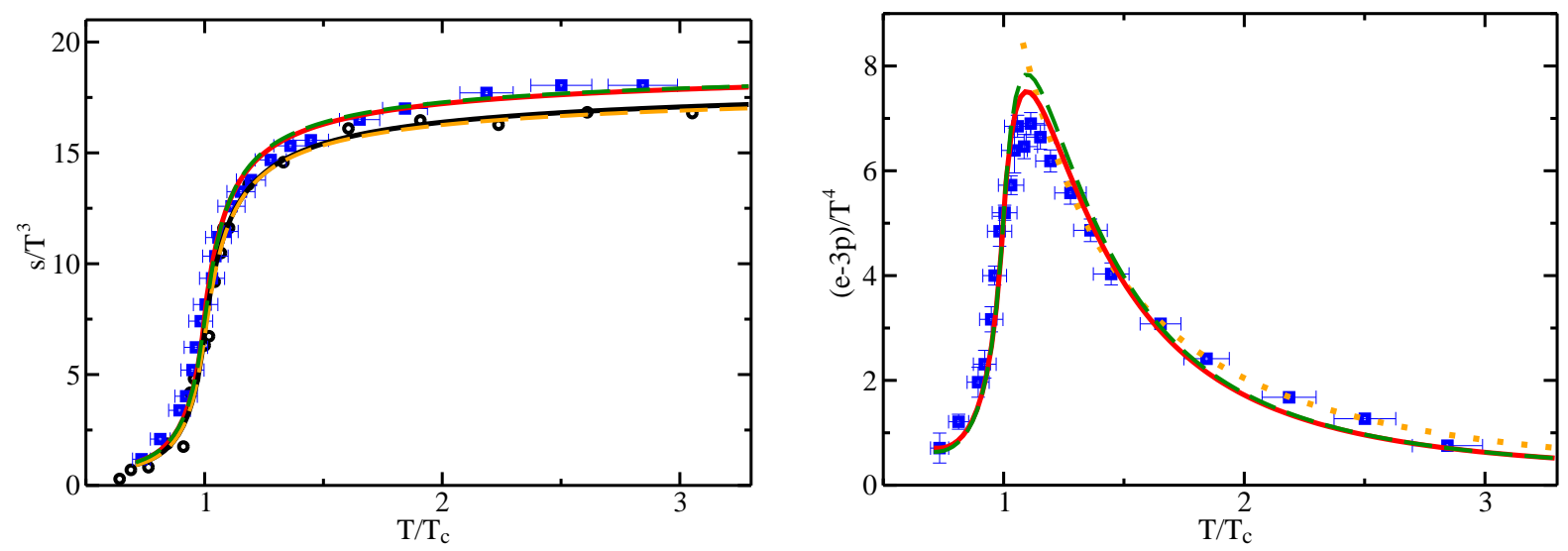

Figure 1: Left: scaled entropy density $s / T^{3}$ as a function of $T / T_{c}$ for $N_{f}=2+1$. Circles [10] and squares [12] exhibit lattice QCD results for different quark mass set-ups. Dashed and solid curves depict QPM results for Fit 1 and Fit 2, respectively, employing different approximations for the quasiparticle dispersion relations as defined in Sec. 2, Lower curves represent adjustments to [10] while upper curves represent quark mass extrapolations in line with [12]. Right: scaled interaction measure $\Delta / T^{4} \equiv(e-3 p) / T^{4}$ as a function of $T / T_{c}$. Squares denote lattice QCD results from [12, 13], dashed and solid curves corresponding QPM results for Fit 1 and Fit 2, respectively. Dotted curve depicts the fuzzy bag model [36] result for $T>1.08 T_{c}$.

quark mass extrapolation to [12]. This highlights the crucial role of the bi-linear term for the quark mass dependence of the phenomenological quasiparticle dispersion relations. For larger $\alpha, G^{2}$ has to take a smaller value at fixed $T$ for describing [10]. However, $\alpha$ cannot be chosen arbitrarily large, as a dominance of $\alpha m_{q} m_{f}$ might lead to an overestimation of the lattice QCD results [12] when extrapolating to the according quark mass values. We mention that using, instead, constant quark mass parameters $m_{u}=m_{d}=0 \mathrm{MeV}$ and $m_{s}=55-90 \mathrm{MeV}$ (which might be considered as physical limit) does not noticeably change the upper dashed curve on the scale exhibited in the left panel of Fig. 1.

In the case of Fit 2, i. e. Eqs. (15) - (7) as ansatz for the self-energies, we first note that the thermal gluon mass increases with decreasing $m_{q}$ according to Eq. (5), while the behavior of the thermal quark mass is, in general, influenced by two counter-acting effects: $M_{+}$increases with decreasing $m_{q}$ whereas the terms solely $\propto m_{q}$ decrease. Thus, depending on an appropriate choice for $\alpha, \Pi_{q}$ might decrease with decreasing $m_{q}$ mostly as a result of $\alpha m_{q} M_{+}$. Consequently, a larger value for $\alpha$ has to be expected compared to Fit 1 in order to saturate the quark mass behavior of $s / T^{3}$ from [12]. Here, for example, we choose $\alpha=2$. The corresponding QPM parameters, being similar to Fit 1 , read $T_{s}=0.68 T_{c}, \lambda=4.77$, $a=-426$ and $b=403.3$ when adjusting to $s / T^{3}$ from [10], cf. lower solid curve in Fig. 1] (left panel). The extrapolation to smaller quark masses in line with [12] is exhibited by the upper solid curve in the left panel of Fig. 1. In both cases, Fit 1 and Fit 2 (on the given scale solid and dashed curves lie almost on top of each other in Fig. 1- left panel), agreement with the corresponding lattice QCD results is found.

In addition, in [12, 13, the scaled interaction measure $\Delta / T^{4}$ was calculated. The QPM results, according to Fit 1 and Fit 2 for the small quark mass set-up, are depicted by dashed and solid curves in the right panel of Fig. 1, respectively, finding an overall good agreement. The necessary pressure integration constant [15], denoted by $B\left(T_{c}\right)$, reads $B\left(T_{c}\right)=0.65 T_{c}^{4}$. Nonetheless, the QPM $m_{q}$ extrapolations overshoot somewhat the lattice QCD results around $T_{c}$ (Fit 1 more than Fit 2) and exhibit small deviations also at larger temperatures. 
Contrary, in a fuzzy bag model approach [36], lattice QCD results of $\Delta / T^{4}$ for $T>1.2 T_{c}$ can perfectly be described, cf. dotted curve in Fig. 1 (right panel). The fuzzy bag picture originally accounts accurately for the plateau observed in the scaled interaction measure $\Delta / T^{2}$ as a function of $T$ (cf. dotted curve in Fig. 2 - left panel), which is parametrized by $\Delta / T^{2}=2 B_{f}+4 B_{M I T} / T^{2}$. Considering leading non-perturbative contributions to the pressure to be given by a temperature dependent (fuzzy) bag constant $B_{f}$ mimicking a gradual rather than an abrupt transition from the confined phase to a nearly perturbative phase, this picture represents a generalization of the MIT bag model. The fit parameters reproducing $\Delta / T^{4}$ for $T>1.2 T_{c}$ from [12, 13] in Fig. 1] read $B_{f}=0.135 \mathrm{GeV}^{2}, B_{M I T}=0.0009 \mathrm{GeV}^{4}$. Nonetheless, this approach neither describes the behavior of the interaction measure in the transition region nor incorporates explicitly quark mass effects.

\section{Scaling properties of the equation of state}

Discussing the scaling properties of the QPM EoS with $m_{q}$ and the value of $T_{c}$, one first notes that the pressure reformulated in a dimensionless fashion reads

$$
\begin{gathered}
\Phi(\xi) \equiv \frac{p(T)}{T^{4}}=\sum_{i=g, q} \epsilon_{i} \frac{d_{i}}{\pi^{2}} \int_{0}^{\infty} d x x^{2} \ln \left(1+\epsilon_{i} e^{-\sqrt{x^{2}+\tilde{\Pi}_{i}}}\right)-b_{0} \xi^{-4} \\
+\sum_{i=g, q} \frac{d_{i}}{2 \pi^{2} \xi^{4}} \int_{1}^{\xi}\left(2 \tilde{\Pi}_{i}^{\prime}+\eta \frac{\partial \tilde{\Pi}_{i}^{\prime}}{\partial \eta}\right) \eta^{3}\left(\int_{0}^{\infty} \frac{d x x^{2}}{\sqrt{x^{2}+\tilde{\Pi}_{i}^{\prime}}} \frac{1}{e^{\sqrt{x^{2}+\tilde{\Pi}_{i}^{\prime}}+\epsilon_{i}}}\right) d \eta
\end{gathered}
$$

with $\tilde{\Pi}_{i}=\Pi_{i}(T) / T^{2}, \tilde{\Pi}_{i}^{\prime}=\Pi_{i}\left(T^{\prime}\right) / T^{\prime 2}$, real number $b_{0}=B\left(T_{c}\right) / T_{c}^{4}$ and $\xi \equiv T / T_{c}, \eta \equiv T^{\prime} / T_{c}$. Eq. (8) depends explicitly on $m_{q}$ via $\Pi_{i}$. For $m_{q} \equiv \epsilon T$ as used in [10], $\tilde{\Pi}_{i}\left(\tilde{\Pi}_{i}^{\prime}\right)$ is a function of $\xi(\eta)$ only because the entering $G^{2}$ depends on $T_{c}$ only via $\xi(\eta)$ (note that $\lambda T_{s} / T_{c}$ is a parameter in the QPM). Thus, $\Phi(\xi)$ displayed as a function of $\xi$ is independent of the explicit value of $T_{c}$. Consequently, related thermodynamic quantities like $s / T^{3}$ and $\Delta / T^{4}$ show the same independence of $T_{c}$. Nonetheless, approximating $m_{q}$ as advocated above in line with [11, 12], $\tilde{\Pi}_{q}$ explicitly depends on $T$, and thus, $\Phi(\xi)$ does not exhibit the discussed $T_{c}$ independence. However, numerically even a variation in $T_{c}$ by 100 $\mathrm{MeV}$ turns out to imply negligible effects on $p / T^{4}, s / T^{3}$ or $\Delta / T^{4}$.

The interaction measure scaled by $T^{2}$ and depicted as a function of $T$ shows a $T_{c}$ dependence, cf. Fig. 2 (left panel). A shift in $T_{c}$ by $5(-5) \mathrm{MeV}$ in line with [12] results in a shift of the curve $\Delta / T^{2}$ to the right (left) in the transition region. In addition, the curves suffer a parallel shift up (down) by about $6-7 \%$ for larger temperatures. Note that the deviations in $\Delta / T^{4}$ observed in Fig. 1 between QPM and [12, 13] for larger $T$ are transparently quantified by deviations from the plateau behavior in Fig. 2 (left panel).

The parallel shift in $\Delta / T^{2}$ can qualitatively be understood from the scaling behavior of the EoS with $T_{c}$. Reformulating $p=T_{c}^{4} \xi\left(\Phi \xi^{3}\right)$ and imposing $\Phi(\xi)=\Phi\left(\xi^{\prime}\right)$, cf. Eq. (8) $), p$ and thus $e=-p+\xi \partial p / \partial \xi$ change when changing $T_{c}$ to $T_{c}^{\prime}$ (i. e. $\xi$ to $\xi^{\prime}$ ) according to $p^{\prime}=p\left(T_{c}^{\prime} / T_{c}\right)^{4}$ and $e^{\prime}=e\left(T_{c}^{\prime} / T_{c}\right)^{4}$. For the EoS in the form $p(e)$, this implies that the linear section at larger $e$, which can be approximated by $p(e)=\alpha e+p_{0}$, becomes $p^{\prime}\left(e^{\prime}\right)=\alpha e^{\prime}+\left(T_{c}^{\prime} / T_{c}\right)^{4} p_{0}$ with the same slope $\alpha$ but different off-set $p_{0}^{\prime}=\left(T_{c}^{\prime} / T_{c}\right)^{4} p_{0}$. For $T_{c}^{\prime}>T_{c}$, the linear section of $p(e)$ is, thus, parallely shifted downward whereas for $T_{c}^{\prime}<T_{c}$ it is shifted upward. A similar behavior is observed for the EoS in the form $p / e$ as exhibited in Fig. 2 (right panel).

Other regions of $p(e)$ might need to be approximated differently, say for instance, by $p(e)=\tilde{\alpha} \sqrt{e}+\tilde{p_{0}}$ close to the transition region. Changing again $T_{c}$ to $T_{c}^{\prime}$, the EoS changes into $p^{\prime}\left(e^{\prime}\right)=\tilde{\alpha}^{\prime} \sqrt{e^{\prime}}+\tilde{p}_{0}^{\prime}$ with

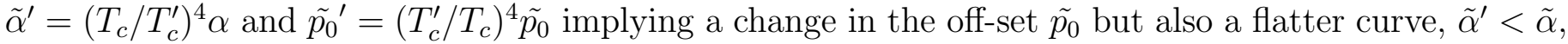
for $T_{c}^{\prime}>T_{c}$ and a steeper curve, $\tilde{\alpha}^{\prime}>\tilde{\alpha}$, for $T_{c}^{\prime}<T_{c}$. This leads, now for $p / e$, to a change in the order of curves as evident from Fig. 2 (right panel) at lower energy densities. 

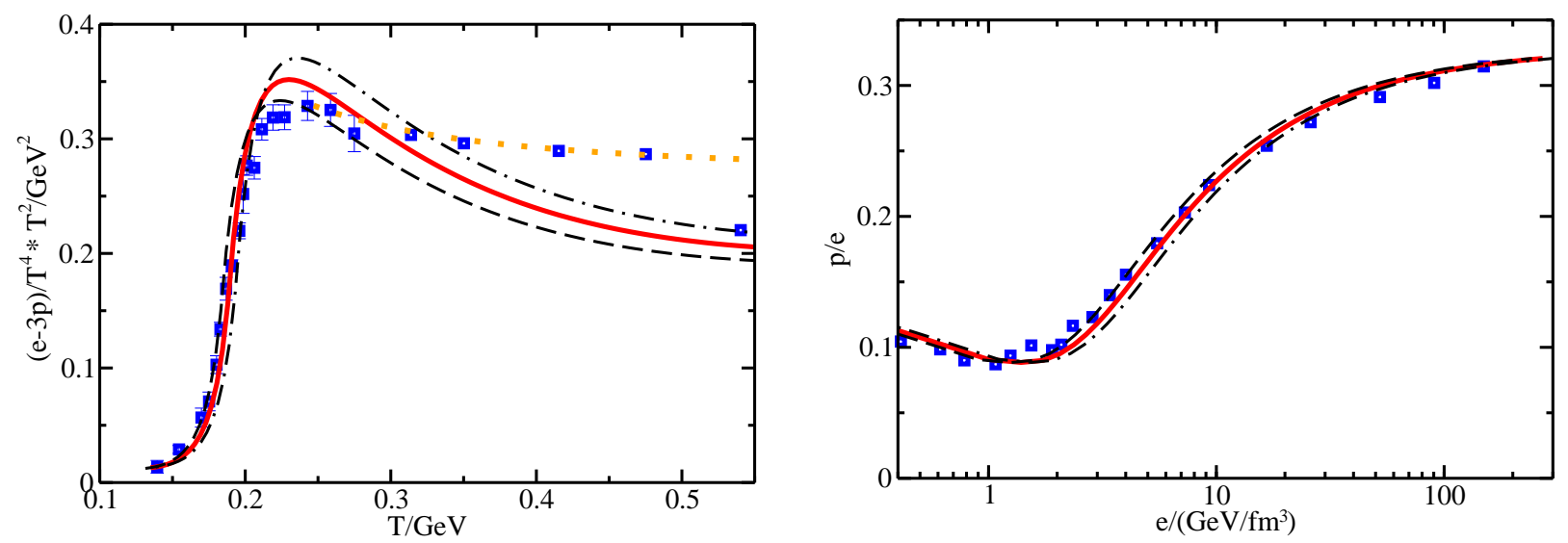

Figure 2: Left: scaled interaction measure $\left(\Delta / T^{2}\right) / G e V^{2}$ as a function of $\mathrm{T} / \mathrm{GeV}$. Squares exhibit lattice QCD results from [12, 13]. Dashed, solid and dash-dotted curves depict QPM results according to Fit 2 using $T_{c}=185,190,195 \mathrm{MeV}$, respectively. Dotted curve shows the directly fitted fuzzy bag model [36] result. Right: EoS in the form $p / e$ as a function of $e /\left(\mathrm{GeV} / \mathrm{fm}^{3}\right)$ and its $T_{c}$ dependence. Lattice QCD results (squares) from [37], curves depict QPM results with the same line code as in the left panel.

Likewise, one may discuss the $m_{q}$ dependence of the EoS. As empirically evident from Fig. 1, the $m_{q}$ dependence of $s / T^{3}$ can be parametrized by $s^{\prime} / T^{3}=\tilde{m}(\xi) s / T^{3}$ with $T$ dependent function $\tilde{m}$. Similarly, $p$ and $e$ (as discussed in [32]) behave according to $p^{\prime}=m(\xi) p$ and $e^{\prime}=m(\xi) e+\xi p \partial m(\xi) / \partial \xi$ with $m_{q}$. Numerically, one finds $m(\xi)=1.107 \ldots 1.081$ in the interval $\xi=1.5 \ldots 3$, i. e. small changes in $m(\xi)$ with $\xi$, whereas for small $\xi$ a larger $m(0.8)=1.33$ and $m(0.9)=1.51$ is found. Thus, $m(\xi)$ varies more sizeably with $\xi$ for small $\xi$. Approximating $p(e)$ again by a linear function, $p^{\prime}=$ $\alpha e^{\prime} /\left(1+\frac{\alpha \xi}{m} \frac{\partial m(\xi)}{\partial \xi}\right)+p_{0} m /\left(1+\frac{\alpha \xi}{m} \frac{\partial m(\xi)}{\partial \xi}\right)$ is found. Consequently, changes in $p(e)$ with $m_{q}$ are negligible for larger $\xi$, where $m(\xi) \approx 1$ is almost constant, whereas they are mostly visible in a region of $\xi$, where $m(\xi)$ varies most rapidly, i. e. for $\xi \leq 1$.

\section{$5 \quad$ Equation of state at asymptotic temperatures}

Recently [28], the pressure for pure SU(3) gauge theory became available in a large temperature interval between $T_{c}$ and $10^{7} T_{c}$ confirming old results [38] between $T_{c}$ and $5 T_{c}$. As $p$ changes most rapidly in the transition region, we fit the QPM parameters to [38] and then continue to larger $T$. The result for $p / p^{S B}$, where $p^{S B}$ denotes the Stefan-Boltzmann pressure, is exhibited by the solid curve in Fig. 3 (left panel). While the QPM describes appropriately the regions around $T_{c}$ and at asymptotically large temperatures, say for $T>200 T_{c}$, it underestimates [28] in the intermediate $T$ region between $3 T_{c}$ and $200 T_{c}$. Although one might find an exotic QPM parametrization for intermediate and large $T$ reproducing [28] and delivering similar results as perturbative QCD [30, 31], cf. dashed curve in Fig. 3 (left panel), such a description fails for $T<2 T_{c}$. This discrepancy might be viewed as a hint for missing degrees of freedom in the intermediate $T$ region. Considering a hard-thermal-loop inspired extension of the QPM [40], including in addition longitudinal gluon (plasmon) and Landau damping contributions, the situation observed in the left panel of Fig. 3 is not measurably improved. This is due to the fact that the effect of a negative partial plasmon pressure decreasing for $T \rightarrow T_{c}^{+}$is compensated by the interwoven increasing partial transverse gluon pressure (driven by the Landau damping) for $T \rightarrow T_{c}^{+}$, while the plasmon contribution vanishes for increasing $T$. Nonetheless, to resolve this issue, additional lattice QCD studies would be desirable. 

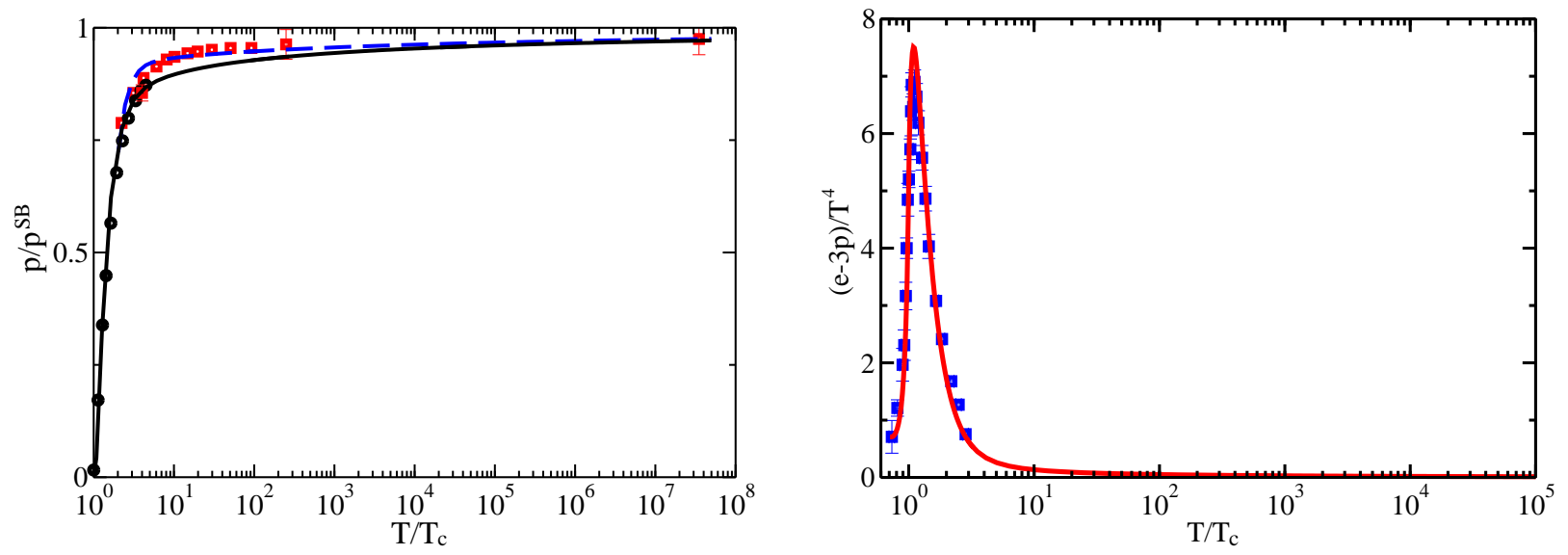

Figure 3: Left: comparison of QPM (solid and dashed curves) with lattice QCD results (circles [38] and squares [28]) for $p / p^{S B}$ as a function of $T / T_{c} \geq 1$ of a pure gluon gas. The QPM is adjusted to [38] with $T_{s}=0.855 T_{c}, \lambda=7, B\left(T_{c}\right)=-0.03 T_{c}^{4}$ (solid curve) or to [28] with $T_{s}=0.97 T_{c}, \lambda=150, B\left(T_{c}\right)=5 T_{c}^{4}$ (dashed curve), where $T_{c}=271 \mathrm{MeV}$ [39]. Dashed curve is similar to perturbative QCD results reported in [30, 31]. Right: scaled interaction measure (Fit 2 in Fig. 1, right panel) as a function of $T / T_{c}$ continued to larger $T$.

Turning to $N_{f}=2+1$ at asymptotic temperatures, the continuation of the scaled interaction measure (cf. Fit 2 in Fig. 1 - right panel) to larger $T$ is exhibited in Fig. 3 (right panel). For large $T$, $\Delta / T^{4}$ approaches zero logarithmically in line with the temperature dependence of $G^{2}$. While already for $T \sim 10 T_{c}$ the conformal limit $e=3 p$ is approximately reached, $p / T^{4}$ still exhibits deviations from $p^{S B} / T^{4}$ of about $10 \%$ at $T=50 T_{c}$ and of $4.5 \%$ at $T=10^{5} T_{c}$. As the dynamically generated thermal gluon and quark masses exhibit a behavior $\sim G T$ in the QPM, they are of the order of $12 \mathrm{TeV}$ and 8 $\mathrm{TeV}$, respectively, at $T=10^{5} T_{c}$, invalidating the naive picture of weakly coupled quarks and gluons with negligible masses.

\section{Conclusion}

In summary, we study the quark mass and temperature dependence of the QCD equation of state. We utilize a quasiparticle model by employing two different expressions for the quasiparticle dispersion relations, which explicitly depend on $m_{q}$ and are based on one-loop QCD approximations [27]. In both cases remarkable agreement with first-principle lattice QCD results is achieved when extrapolating in the $m_{q}$ parameter space. Scaling properties of the QCD EoS with $m_{q}$ and $T_{c}$ are discussed and the EoS is continued to asymptotically large $T$, where very hot QCD matter may be viewed as composed of rather heavy quasiparticle excitations.

The authors thank E. Laermann and Z. Fodor for valuable discussions. The work is supported by BMBF 06DR136 and EU I3HP.

\section{References}

[1] C. Höhne (NA49 Collaboration), Nucl. Phys. A 774 (2006) 35

[2] The First Three Years of Operation of RHIC, Nucl. Phys. A 757 (2005) 1

[3] U. Heinz, Nucl. Phys. A 721 (2003) 30 
[4] E. V. Shuryak, Prog. Part. Nucl. Phys. 53 (2004) 273

[5] E. V. Shuryak, Nucl. Phys. A 750 (2005) 64

[6] M. Gyulassy, and L. D. McLerran, Nucl. Phys. A 750 (2005) 30

[7] D. Teaney, J. Lauret, and E. V. Shuryak, Phys. Rev. Lett. 86 (2001) 4783

[8] P. F. Kolb, P. Huovinen, U. Heinz, and H. Heiselberg, Phys. Lett. B 500 (2001) 232

[9] L. P. Csernai, J. I. Kapusta, and L. D. McLerran, Phys. Rev. Lett. 97 (2006) 152303

[10] F. Karsch, K. Redlich, and A. Tawfik, Eur. Phys. J. C 29 (2003) 549

[11] M. Cheng et al., Phys. Rev. D 77 (2008) 014511

[12] R. Gupta (HotQCD Collaboration), preprint arXiv:0810.1764 [hep-lat]

[13] W. Söldner (RBC-Bielefeld and HotQCD Collaborations), preprint arXiv:0810.2468 [hep-lat]

[14] A. Peshier, B. Kämpfer, O. P. Pavlenko, and G. Soff, Phys. Lett. B 337 (1994) 235, Phys. Rev. D 54 (1996) 2399 ; A. Peshier, B. Kämpfer, and G. Soff, Phys. Rev. C 61 (2000) 045203, Phys. Rev. D 66 (2002) 094003

[15] M. Bluhm, B. Kämpfer, and G. Soff, Phys. Lett. B 620 (2005) 131 ; M. Bluhm et al., Phys. Rev. C 76 (2007) 034901 ; M. Bluhm, B. Kämpfer, R. Schulze, and D. Seipt, Eur. Phys. J. C 49 (2007) 205

[16] T. S. Biro, P. Levai, P. Van, and J. Zimanyi, Phys. Rev. C 75 (2007) 034910

[17] Y. B. Ivanov et al., Phys. Rev. C 72 (2005) 025804

[18] A. S. Khvorostukin, V. V. Skokov, V. D. Toneev, and K. Redlich, Eur. Phys. J. C 48 (2006) 531

[19] W. Cassing, Nucl. Phys. A 791 (2007) 365, Nucl. Phys. A 795 (2007) 70

[20] C. Ratti, M. A. Thaler, and W. Weise, Phys. Rev. D 73 (2006) 014019

[21] S. Rößner, C. Ratti, and W. Weise, Phys. Rev. D 75 (2007) 034007

[22] S. Mukherjee, M. G. Mustafa, and R. Ray, Phys. Rev. D 75 (2007) 094015

[23] B.-J. Schäfer, and J. Wambach, Nucl. Phys. A 757 (2005) 479

[24] B.-J. Schäfer, J. M. Pawlowski, and J. Wambach, Phys. Rev. D 76 (2007) 074023

[25] E. V. Shuryak, and I. Zahed, Phys. Rev. D 70 (2004) 054507

[26] B. A. Gelman, E. V. Shuryak, and I. Zahed, Phys. Rev. C 74 (2006) 044908, Phys. Rev. C 74 (2006) 044909

[27] D. Seipt, M. Bluhm, and B. Kämpfer, preprint arXiv:0810.3803 [hep-ph]

[28] G. Endrödi, Z. Fodor, S. D. Katz, and K. K. Szabo, Proc. Sci. LAT2007 (2007) 228

[29] J.-P. Blaizot, A. Rebhan, and E. Iancu, Phys. Rev. D 63 (2001) 065003

[30] K. Kajantie, M. Laine, K. Rummukainen, and Y. Schröder, Phys. Rev. D 67 (2006) 105008

[31] M. Laine, and Y. Schröder, Phys. Rev. D 73 (2006) 085009

[32] M. Bluhm, and B. Kämpfer, preprint arXiv:0807.4080 [hep-ph]

[33] R. D. Pisarski, Nucl. Phys. A 498 (1989) 423c

[34] C. DeTar, and R. Gupta (HotQCD Collaboration), Proc. Sci. LAT2007 (2007) 179

[35] E. S. Fraga, L. F. Palhares, and C. Villavicencio, preprint arXiv:0810.1060 [hep-ph]

[36] R. D. Pisarski, Prog. Theor. Phys. Suppl. 168 (2007) 276

[37] C. Schmidt (HotQCD Collaboration), preprint arXiv:0810.0374 [hep-lat]

[38] G. Boyd et al., Nucl. Phys. B 469 (1996) 419

[39] F. Karsch, Lect. Notes Phys. 583 (2002) 209

[40] R. Schulze, M. Bluhm, and B. Kämpfer, Eur. Phys. J. ST 155 (2008) 177, preprint arXiv:0803.1571 [hep-ph]; R. Schulze, and B. Kämpfer, preprint arXiv:0811.0274 [hep-ph], these proceedings 